\title{
Influence de la densité de plantation et du mode d'entretien du sol sur l'enracinement d'un peuplement de vigne planté en sol favorable
}

\author{
René MORLAT, Michel REMOUÉ $\left({ }^{*}\right) \&$ Patrice PINET $(* *)$ \\ I.N.R.A., Station d'Agronomie, F 49000 Angers \\ (*) I.N.R.A., Domaine expérimental viticole, F 49260 Montreuil-Bellay

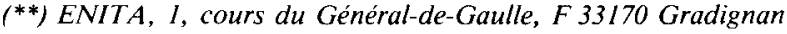

RÉSUMÉ

\begin{abstract}
Dans un essai situé à Montreuil-Bellay (Maine-et-Loire), plusieurs densités de plantation réalisées avec le cépage "Chenin" sont comparées selon 2 modes d'entretien du sol (désherbage chimique, enherbement permanent). Une étude du système racinaire a été faite, dans le but de mieux comprendre les etfets de compétition qui s'exercent sur la partie souterraine. Les grands écartements entre rangs $(2,60 \mathrm{~m})$ augmentent la densité racinaire, tandis que les grands espacements sur la ligne de plantation $(1,50 \mathrm{~m})$ la diminuent. En conséquence, la densité de plantation $1,80 \mathrm{~m} \times 1,50 \mathrm{~m}$ a un effet défavorable sur le système racinaire. L'enherbement permanent fait décroître considérablement le nombre de racines de vigne, principalement au centre de l'interligne. La compétition entre souches pour le risque théorique le plus élevé $(1,80 \mathrm{~m} \times 1,10 \mathrm{~m}) \mathrm{n}$ 'améliore pas le degré d'exploitation du sol. Les vignes larges ayant une vigueur supérieure entraînent une meilleure colonisation du sol.
\end{abstract}

Mots clés additionnels : Structure racinaire, écartement, espacement, compétition, système de conduite.

Effects of planting density and soil cultivation practices on the root system of vine.

In a field experiment, started in 1971 at Montreuil-Bellay (Loire) Valley, several planting densities ("Chenin" vine grafted on SO4) were compared with 2 soil management treatments (chemical weed control and permanent grass cover). Nine years after planting the vineyard, root-counts were made, simultaneously with pedological profile studies. Large row spacing $(2.60 \mathrm{~m})$ increased root density, while large spacing along the row decreased root number. $1.80 \mathrm{~m} \times 1.50 \mathrm{~m}$ spacing had the most unfavourable effect on the vine root system. Permanent grass-cover (rye-grass) led to a marked reduction of roots between rows, and particularly near the soil surface. The best root system development was obtained with low planting densities.

Additional key-words : Root system structure, row spacing, spacing along the row, competition, training system.

\section{INTRODUCTION}

En viticulture, la densité de plantation et le mode d'entretien du sol sont deux facteurs importants qui peuvent modifier profondément le rendement et la qualité. Ces deux paramètres jouent un rôle à différents niveaux du peuplement (partie aérienne, système racinaire, bioclimat). Dans cette note, seul sera envisagé l'effet sur le système racinaire.

Les travaux concernant l'action de la densité de plantation sur le système racinaire sont assez rares (HIDALGO \& CANDELA, 1969; LAURENT, 1979; RADOULOV \& BRAIKOV, 1974 ; SCHATOUIRO et al., 1978 ; BRANAS \& VERGNES, 1957).
Les conclusions de ces études ne sont pas toujours claires et parfois même contradictoires. A notre connaissance, l'expérimentation la plus complète est celle d'HIDALGO \& CANDELA, mais les résultats sont obtenus, soit pour des densités différentes avec des plantations au carré, soit pour une même densité avec plusieurs écartements entre rangs et espacements sur le rang. Grâce à l'essai de Montreuil-Bellay, nous pouvons étudier 6 densités de plantation différentes obtenues par variation de l'écartement et de l'espacement ; cette étude a été financée par l'ATP " compétition intraclonale chez les végétaux ligneux » dont le responsable était J. HUET. 


\section{MATÉRIEL ET MÉTHODES}

Pour l'étude du système racinaire, nous avons retenu seulement 4 densités de plantation qui combinent 2 espacements sur le rang $(1,10 \mathrm{~m} ; 1,50 \mathrm{~m})$ et 2 écartements entre rangs $(1,80 \mathrm{~m} ; 2,60 \mathrm{~m})$. Ces densités ont été étudiées en désherbage chimique intégral et avec enherbement permanent à base de ray-grass anglais de l'interligne. Une bande de $80 \mathrm{~cm}$ sous la ligne de plantation est maintenue désherbée.

Pour des raisons matérielles, l'espacement de $1,25 \mathrm{~m}$ n'a pas été retenu, de même que le traitement $2,60 \mathrm{~m} \times 1,10 \mathrm{~m}$ enherbé. Cette perte d'information est limitée car il n'y a pas d'interaction mode d'entretien $\times$ écartement, significative.

La vigne, plantée en 1971, est conduite avec une taille Guyot simple palissée et une hauteur de tronc de $60 \mathrm{~cm}$. Le rapport hauteur de feuillage sur écartement entre rangs est maintenu constant de même que la charge par unité de surface. Le cépage choisi est le "Chenin " greffé sur SO4. La fertilisation est identique quel que soit le traitement. Le vignoble est exposé sur une pente Sud, orienté Nord-Sud et planté dans un sol argileux-limoneux issu de l'altération de marnes légèrement pyriteuses (sol brun calcique argilisé, tabl. 1).

TABLEAU 1

Caractéristiques du sol. Soil characteristics.

\begin{tabular}{lccccc}
\hline \hline \multicolumn{1}{c}{ Granulométrie \% } & Argile & $\begin{array}{c}\text { Limon } \\
\text { fin }\end{array}$ & $\begin{array}{c}\text { Limon } \\
\text { grossier }\end{array}$ & $\begin{array}{c}\text { Sable } \\
\text { fin }\end{array}$ & $\begin{array}{c}\text { Sable } \\
\text { grossier }\end{array}$ \\
\hline Horizons & & & & & \\
\hline Ap1 & 28,2 & 16,5 & 12,1 & 16,4 & 26,7 \\
\hline (B) & 29,3 & 16,5 & 11,8 & 15,8 & 26,6 \\
\hline B/C & 35,3 & 17,9 & 12,0 & 13,8 & 21,0 \\
\hline C & 35,5 & 18,4 & 10,9 & 11,8 & 23,4 \\
\hline \hline
\end{tabular}

Le sol est favorable à un bon développement de la vigne, grâce à des propriétés physico-chimiques satisfaisantes et à des réserves hydriques élevées. L'enracinement n'est pas confronté à des contraintes mécaniques brutales comme le montre dans la figure 1 le profil de résistance à la pénétration (mesures au pénétromètre à cône, modèle SOLETANCHE M. \& O.).

La méthode d'étude du système racinaire permet d'annuler l'effet d'une asymétrie éventuelle de l'enracinement par rapport à la ligne de plantation (MORLAT, 1981). Les racines sont comptées par classe de diamètre $(<1 \mathrm{~mm}, 1-2 \mathrm{~mm},>2 \mathrm{~mm})$ et selon les horizons pédologiques, sur les parois de fosses creusées parallèlement à la ligne de ceps. Deux plans de comptage, dont la longueur est égale à la distance de plantation $(1,10 \mathrm{~m}$ ou $1,50 \mathrm{~m})$ sont retenus : l'un sur la ligne, l'autre au milieu de l'interligne. Le système racinaire plongeant a été apprécié en comptant horizontalement sur le plancher des fosses (sur une largeur de $20 \mathrm{~cm}$ ) les sections de racines. Nous avons

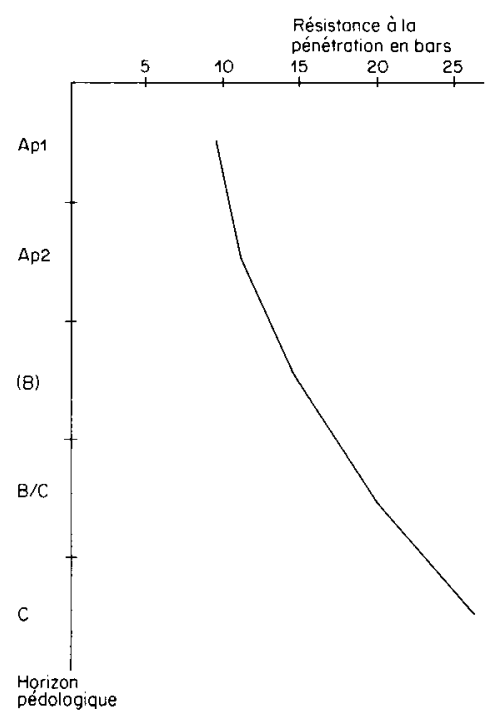

Figure 1

Profil moyen des résistances à la pénétration (ligne + interligne). Mean mechanical resistance of different horizons.

effectué 6 répétitions par traitement. Sous enherbement, l'enracinement du ray-grass a été étudié à partir de prélèvements effectués par carottages. Les racines sont triées par lavages successifs puis pesées après séchage à $105^{\circ} \mathrm{C}$.

Une analyse de variance à plusieurs critères de classification (DAGNELIE, 1975) a été réalisée et les moyennes ont été comparées par le test de NEWMAN \& Keuls. En complément, à partir de la matrice des corrélations (cf. tabl. 9), nous avons réalisé une analyse en composantes principales qui, par rapport à une régression multiple, a l'avantage de pondérer les données et de permettre une représentation graphique en 2 dimensions. Les mesures sont réduites mais non centrées. Cette analyse permet d'étudier les liaisons entre classes de diamètre des racines et, simultanément, les actions combinées des facteurs principaux de l'essai (mode d'entretien du sol, espacement, écartement, distance à la souche).

\section{RÉSULTATS}

\section{A. Etude par l'analyse de variance}

\section{Influence de la densité de plantation}

\section{a) Racines de diamètre inférieur à $1 \mathrm{~mm}$}

Cette classe représente une fraction du système racinaire intervenant pour une large part dans l'alimentation hydrique et minérale de la vigne (MORLAT, 1981). Ces racines sont surtout présentes dans les horizons supérieurs et soumises à de nombreux cycles dessiccation-humectation. Sur les profils étudiés (90 à $110 \mathrm{~cm}$ de hauteur), la densité de plantation n'exerce pas d'influence significative (tabl. 2). Cette classe de diamètre est bien répartie dans le volume exploitable puisque le plan de comptage n'est pas significatif. Les horizons de surface sont les plus 


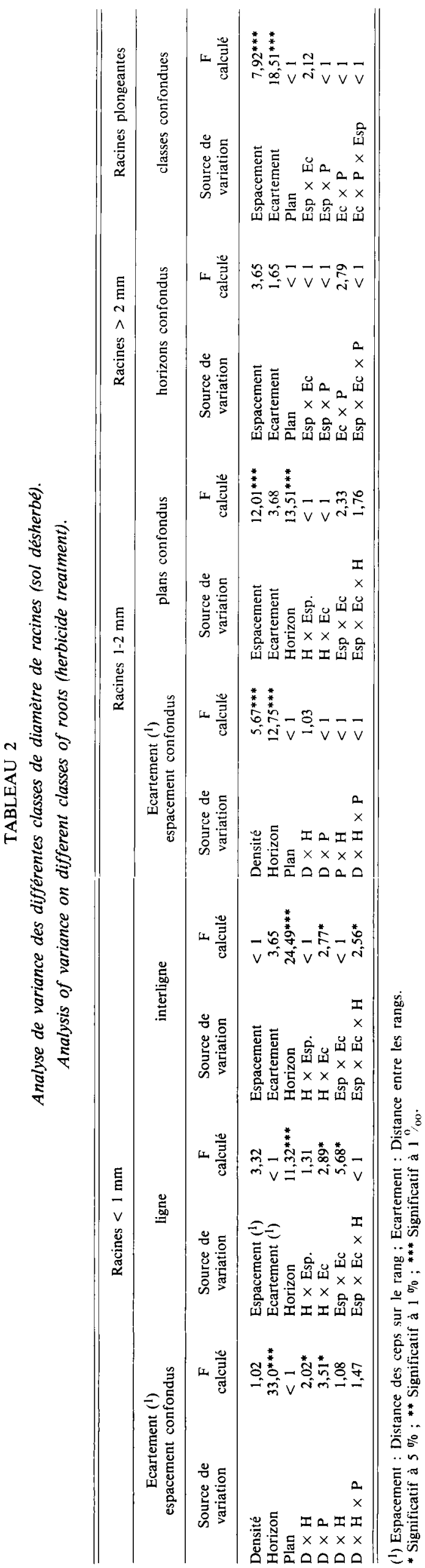

prospectés (tabl. 3). En profondeur (horizon C), le traitement $1,80 \mathrm{~m} \times 1,50 \mathrm{~m}$ entraîne une diminution

TABLEAU 3

Nombre moven de racines de diamètre inférieur à $1 \mathrm{~mm}$ avec désherbage (résultats exprimés en racines $/ \mathrm{m}^{2}$ d'horizon): moyenne de la ligne et interligne.

Mean number of very fine roots $(<1 \mathrm{~mm})$ under the herbicide treatment (in roots $/ \mathrm{m}^{2}$ of horizon) : mean of samples from along and between rows.

\begin{tabular}{lrrrr}
\hline \multicolumn{1}{c}{ Densité } & & & & \\
Horizon & $1,80 \times 1,10$ & $1,80 \times 1,50$ & $2,60 \times 1,10$ & $2,60 \times 1,50$ \\
\hline Ap1 & & & & \\
Ap2 & $198 \pm 37,6$ & $233 \pm 53,6$ & $220 \pm 32,6$ & $217 \pm 37,8$ \\
(B) & $229 \pm 45,6$ & $200 \pm 36,9$ & $210 \pm 44,4$ & $241 \pm 48,6$ \\
B/C & $129 \pm 31,2$ & $114 \pm 26,0$ & $142 \pm 44,4$ & $116 \pm 35,8$ \\
C & $127 \pm 48,4$ & $137 \pm 32,0$ & $130 \pm 23,7$ & $132 \pm 24,2$ \\
& $122 \pm 51,2$ & $77 \pm 19,0$ & $135 \pm 43,0$ & $157 \pm 33,6$ \\
\hline
\end{tabular}

du nombre de racines, confirmée par l'interaction significative densité $\times$ horizon (tabl. 4). L'espacement et l'écartement ne sont pas significatifs, mais l'horizon a un effet très hautement significatif. Certaines interactions sont significatives (tabl. 2). Ainsi, pour un écartement de $1,80 \mathrm{~m}$, un espacement de $1,10 \mathrm{~m}$ augmente le nombre de racines sous la ligne de plantation. Pour un espacement de $1,50 \mathrm{~m}$, la densité racinaire croît avec un écartement de $2,60 \mathrm{~m}$. La densité $1,80 \mathrm{~m} \times 1,50 \mathrm{~m}$ a tendance à provoquer un effet défavorable sur l'enracinement développé sous la ligne de ceps.

\section{TABLEAU 4}

Nombre moyen de racines de la classe inférieure à $1 \mathrm{~mm}$ avec désherbage (résultats exprimés en racines $/ m^{2}$ d'horizon) : ligne et interligne séparées.

Mean number of very fine roots $(<I \mathrm{~mm})$ under the herbicide treatments (roots $/ \mathrm{m}^{2}$ of horizon): results presented separately for samples along and between rows.

\begin{tabular}{llrrrr}
\hline \hline & Densité & & & & \\
Horizon & $1,80 \times 1,10$ & $1,80 \times 1,50$ & $2,60 \times 1,10$ & $2,60 \times 1,50$ \\
Apl & & & & \\
\hline \multirow{2}{*}{ Ap2 } & Ligne & $1,89 \pm 64,7$ & $175 \pm 57,4$ & $202 \pm 50,6$ & $232 \pm 74,9$ \\
& Interligne & $208 \pm 52,0$ & $291 \pm 55,0$ & $240 \pm 43,7$ & $202 \pm 31,8$ \\
& Interligne & $231 \pm 88,1$ & $186 \pm 52,9$ & $230 \pm 80,2$ & $239 \pm 48,6$ \\
(B) & Ligne & $132 \pm 51,8$ & $213 \pm 59,5$ & $191 \pm 50,0$ & $243 \pm 97,4$ \\
& Interligne & $126 \pm 55,0$ & $126 \pm 40,6$ & $114 \pm 47,0$ & $100 \pm 51,6$ \\
B/C C & Ligne & $138 \pm 80,8$ & $136 \pm 37,6$ & $169 \pm 48,5$ & $132 \pm 56,0$ \\
& Interligne & $115 \pm 69,6$ & $139 \pm 62,6$ & $140 \pm 44,6$ & $134 \pm 29,7$ \\
C & Ligne & $156 \pm 91,0$ & $86 \pm 23,4$ & $143 \pm 86,6$ & $130 \pm 45,1$ \\
& Interligne & $89 \pm 49,4$ & $67 \pm 32,1$ & $126 \pm 40,1$ & $178 \pm 27,9$ \\
& & & & & \\
\hline
\end{tabular}

\section{b) Racines de diamètre $1-2 \mathrm{~mm}$}

En sol désherbé, la densité de plantation et l'horizon pédologique ont des effets hautement significatifs, tandis que le plan de comptage est sans influence. L'espacement est très hautement significatif et l'écartement est à la limite de signification (tabl. 2). En surface, les vignes étroites ont un nombre de racines plus élevé, alors que le phénomène s'inverse en profondeur (tabl. 5). 
TABLEAU 5

Nombre moyen de racines de diamètre 1-2 $\mathrm{mm}$ avec désherbage (résultats exprimés en racines $/ \mathrm{m}^{2}$ d'horizon).

Mean number of medium roots $(1-2 \mathrm{~mm})$ under the herbicide treatment (roots $/ \mathrm{m}^{2}$ of horizon).

\begin{tabular}{lllll}
\hline \hline \multirow{2}{*}{ Densité } & & & & \\
Horizon & $1,80 \times 1,10$ & $1,80 \times 1,50$ & $2,60 \times 1,10$ & $2,60 \times 1,50$ \\
\hline Ap1 & & & & \\
Ap2 & $48,5 \pm 34,7$ & $29,5 \pm 19,1$ & $36,5 \pm 19,4$ & $34,0 \pm 19,3$ \\
(B) & $82,0 \pm 25,5$ & $40,0 \pm 15,6$ & $66,0 \pm 19,4$ & $64,0 \pm 19,2$ \\
B/C & $38,0 \pm 16,9$ & $26,0 \pm 12,7$ & $47,0 \pm 13,0$ & $43,0 \pm 19,0$ \\
C & $43,5 \pm 40,2$ & $36,5 \pm 22,4$ & $59,5 \pm 33,4$ & $42,0 \pm 18,5$ \\
\hline \hline
\end{tabular}

\section{c) Racines de diamètre supérieur à $2 \mathrm{~mm}$}

Elles sont généralement réparties dans les horizons Ap2, (B) et C, mais nous avons réalisé l'analyse statistique seulement sur le profil entier. Les effets de l'écartement et du plan de comptage ne sont pas significatifs tandis que l'espacement a une influence proche de la signification (tabl. 2). Les grands espacements sur la ligne $(1,50 \mathrm{~m})$ diminuent le nombre de racines (tabl. 6).

\section{TABLEAU 6}

Nombre moyen de racines de diamètre supérieur à $2 \mathrm{~mm}$ avec désherbage (résultats exprimés en racines $/ m^{2}$ d'horizon). Mean number of large roots ( $>2 \mathrm{~mm}$ )

under the herbicide treatment (roots $/ \mathrm{m}^{2}$ of horizon).

Densité

Horizon

$1,80 \times 1,10 \quad 1,80 \times 1,50 \quad 2,60 \times 1,10 \quad 2,60 \times 1,50$

\begin{tabular}{lrrrr}
\hline Ap1 & $8,0 \pm 18,0$ & $12,7 \pm 13,4$ & $7,0 \pm 8,0$ & $11,0 \pm 5,8$ \\
Ap2 & $59,0 \pm 34,6$ & $36,7 \pm 9,8$ & $50,0 \pm 14,6$ & $54,0 \pm 13,2$ \\
(B) & $28,0 \pm 29,6$ & $18,0 \pm 13,6$ & $36,5 \pm 30,0$ & $22,5 \pm 16,8$ \\
B/C & $13,0 \pm 16,5$ & $13,0 \pm 16,0$ & $28,0 \pm 18,0$ & $14,0 \pm 9,9$ \\
C & $10,0 \pm 10,0$ & $8,0 \pm 5,7$ & $12,0 \pm 6,8$ & $8,5 \pm 5,5$ \\
\hline
\end{tabular}

\section{d) Racines plongeantes}

Leur comptage permet d'obtenir une certaine image du système racinaire développé dans le matériau géologique (marne). L'analyse statistique a été réalisée à partir des classes regroupées, car les mêmes phénomènes s'observent quel que soit le diamètre (tabl. 7). L'espacement et l'écartement ont des effets hautement significatifs alors que le plan de comptage n'intervient pas. Les grands écartements $(2,60 \mathrm{~m})$ accroissent le nombre de racines, tandis que les forts espacements le diminuent. En conséquence, la densité $1,80 \mathrm{~m} \times 1,50 \mathrm{~m}$ possède le plus mauvais système racinaire plongeant. Les racines de diamètre inférieur à $1 \mathrm{~mm}$ sont plus nombreuses dans les vignes larges (tabl. 7).

\section{Influence du mode d'entretien du sol}

Pour une densité de plantation donnée $(1,80 \mathrm{~m} \times 1,10 \mathrm{~m})$, l'horizon, le mode d'entretien du sol et le plan de comptage sont hautement significatifs en ce qui concerne les racines de diamètre infé-

\section{TABLEAU 7}

Nombre moyen de racines plongeantes selon la classe de diamètre avec désherbage (résultats exprimés en racines $/ \mathrm{m}^{2}$ ).

Mean number of deep roots of various diameter classes under the herbicide treatment (roots $/ \mathrm{m}^{2}$ ).

\begin{tabular}{lrrrrr}
\hline \hline Densité & & & & \\
Diamètre de & $1,80 \times 1,10$ & $1,80 \times 1,50$ & $2,60 \times 1,10$ & $2.60 \times 1,50$ \\
racines & & & & \\
\hline$\varnothing<$ & $1 \mathrm{~mm}$ & $76,0 \pm 60,8$ & $53,0 \pm 44,0$ & $161,0 \pm 11,0$ & $106,0 \pm 34,7$ \\
$\varnothing$ & $1-2 \mathrm{~mm}$ & $19,0 \pm 11,8$ & $14,0 \pm 7,8$ & $42,5 \pm 15,6$ & $21,0 \pm 11,3$ \\
$\varnothing>$ & $2 \mathrm{~mm}$ & $8,0 \pm 9,5$ & $7,0 \pm 5,3$ & $24,0 \pm 17,0$ & $8,0 \pm 6,0$ \\
\hline
\end{tabular}

rieur à $1 \mathrm{~mm}$ (tabl. 8). L'enherbement permanent diminue leur nombre au centre de l'interligne et particulièrement en surface.

Pour un espacement de $1,50 \mathrm{~m}$, il apparaît une interaction plan de comptage $\times$ mode d'entretien du sol. Le mode de culture du sol n'a pas d'influence sur le nombre de racines de diamètre 1-2 $\mathrm{mm}$. Par contre, l'enherbement permanent diminue significativement le nombre de racines de diamètre supérieur à $2 \mathrm{~mm}$. L'effet du mode d'entretien du sol est proche de la limite de signification pour le système racinaire plongeant et se manifeste par une tendance à l'accroissement du nombre de racines avec l'enherbement. Des résultats parallèles ont déjà été obtenus dans d'autres essais (MORLAT, 1981).

En surface, le nombre de racines de diamètre inférieur à $1 \mathrm{~mm}$ est fortement corrélé négativement $\left(\mathrm{r}=-0,89^{* * *}\right)$ au poids de racines de ray-grass (PINET, 1980).

\section{Résultats concernant l'asymétrie de l'enracinement}

Cette caractéristique du système racinaire permet une certaine quantification de la distribution de l'enracinement dans le volume disponible de part et d'autre de la souche. Elle s'exprime par l'indice (I)

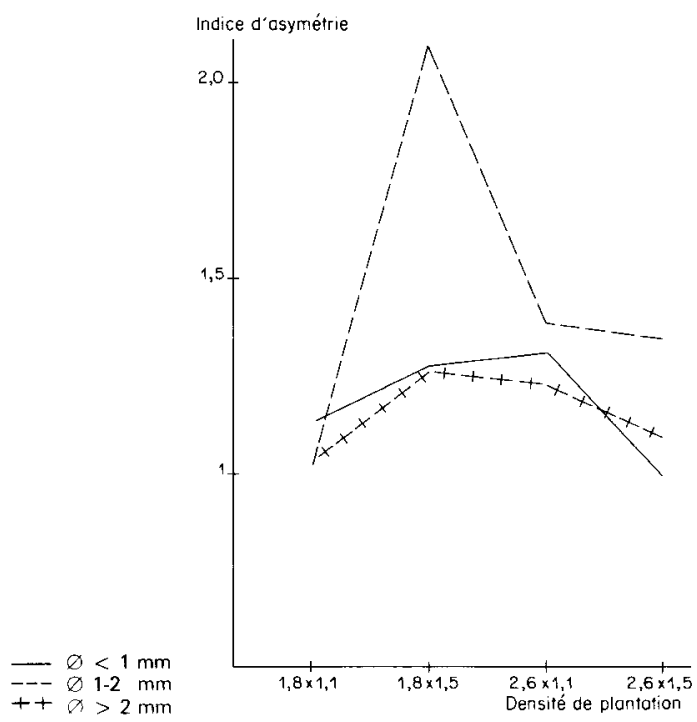

Figure 2

Indice d'asymétrie du système racinaire (résultats obtenus pour l'interligne et l'ensemble du profill.

Root asymetric index (results obtained on inter-rows and whole profile). 


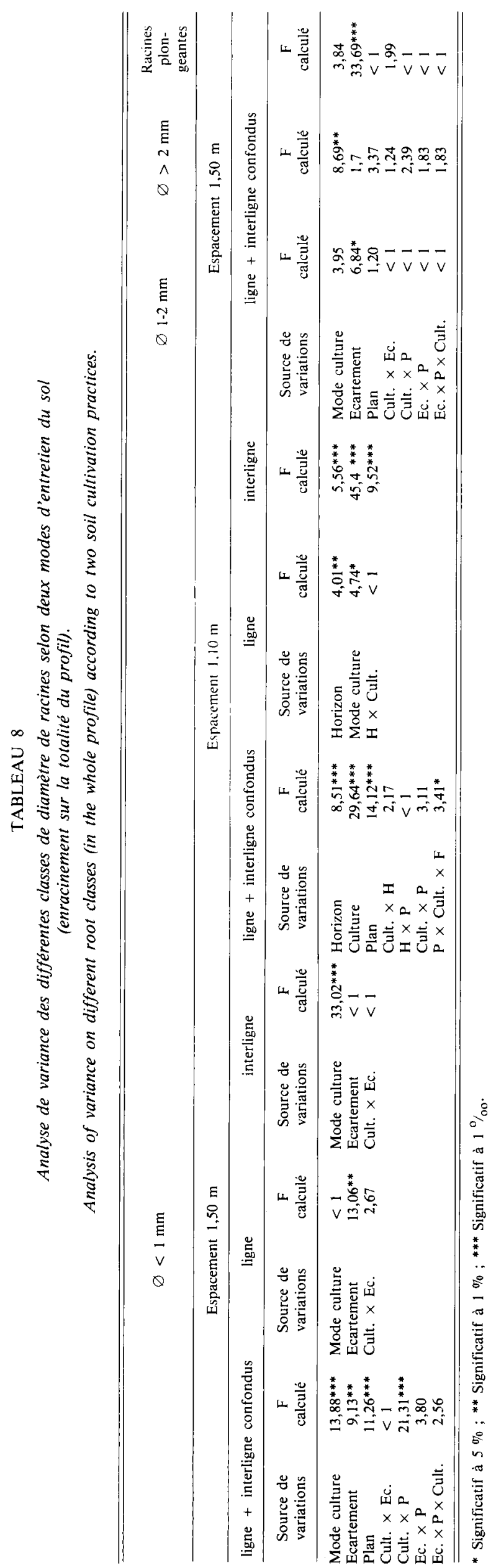

défini comme le rapport des moyennes partielles obtenues à partir des fosses d'observation situées en positions opposées par rapport à la ligne de plantation (MORLAT, 1981).

Les effets de la densité de plantation sont sensibles sur la classe de diamètre 1-2 mm. Les fortes densités $(1,80 \mathrm{~m} \times 1,10 \mathrm{~m})$ ont dans l'ensemble l'action la plus favorable puisque l'indice d'asymétrie est voisin de 1. Par contre, la densité de plantation $1,80 \mathrm{~m} \times 1,50 \mathrm{~m}$ entraîne une grande hétérogénéité dans la répartition des racines spécialement avec la classe 1-2 $\mathrm{mm}$ pour laquelle l'indice est supérieur à 2 (fig. 2).

\section{B. Etude au moyen de l'analyse multidimensionnelle}

Le traitement des données (nombres de racines $/ \mathrm{m}^{2}$ sur l'ensemble de la paroi de comptage) par l'analyse en composantes principales fait apparaître une faible corrélation entre les classes de diamètre (tabl. 9).

TABLEAU 9

Matrice des corrélations entre les groupes de diamètres de racines. Correlation between different root diameter classes.

\begin{tabular}{cccc}
\hline \hline Classes de diamètre & $\varnothing<1 \mathrm{~mm}$ & $\varnothing 1-2 \mathrm{~mm}$ & $\varnothing>2 \mathrm{~mm}$ \\
\hline$\varnothing<1 \mathrm{~mm}$ & 1 & 0,5619 & 0,3037 \\
\hline$\varnothing 1-2 \mathrm{~mm}$ & & 1 & 0,4471 \\
\hline$\varnothing>2 \mathrm{~mm}$ & & & 1 \\
\hline
\end{tabular}

- L'axe 1 (tabl. 10) expliquant 62,8 p. 100 de la variation, traduit bien l'importance de l'ensemble de l'enracinement. La valeur de la projection sur l'axe représente la somme des valeurs réduites des 3 classes de diamètre pour un traitement quelconque, lesquelles ont une contribution sensiblement égale (vecteur propre : 0,$82 ; 1 ; 0,92$ ).

TABLEAU 10

Tableau des composantes et matrice des vecteurs propres normés correspondants.

Components and matrix of normal vectors.

\begin{tabular}{lllll}
\hline \hline & & $\lambda 1$ & $\lambda 2$ & $\lambda 3$ \\
\hline \multirow{2}{*}{ Racines } & Valeur & 1,884 & 0,708 & 0,409 \\
& $\%$ variation & 62,8 & 23,6 & 13,6 \\
\hline \multirow{2}{*}{$\begin{array}{c}\text { Vecteurs, } \\
\text { propres } \\
\text { normés }\end{array}$} & $\varnothing<1 \mathrm{~mm}$ & 0,822 & 1 & $-0,364$ \\
\cline { 2 - 5 } & $\varnothing>2 \mathrm{~mm}$ & 1 & $-0,174$ & 1 \\
\hline \hline
\end{tabular}

- L'axe 2 (23,6 p. 100 de la variation) traduit l'opposition entre la fraction racinaire absorbante (tabl. 10) et la charpente (vecteur propre: 1 ; $-0,17 ;-0,705)$. 
- La lecture de la figure 3 fait apparaître clairement dans les projections sur l'axe 1, l'influence défavorable de l'enherbement sur le système racinaire. Sur l'axe 2, l'enherbement provoque un déséquilibre très marqué (surtout avec les faibles écartements) entre l'interligne et la ligne. De même, sur l'axe 1 , le nombre de racines des 3 classes augmente avec la non culture, les grands écartements et les faibles espacements.

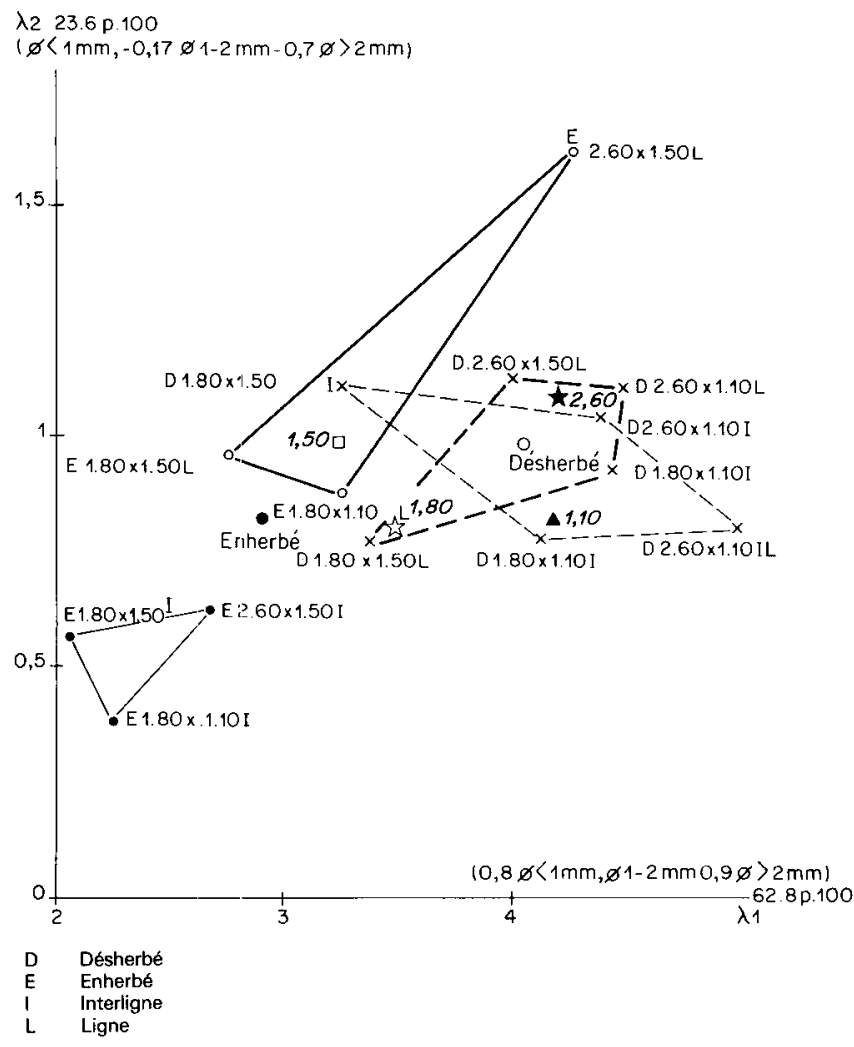

Figure 3

Analyse en composantes principales. Projections sur le premier plan principal.

Factor analysis and projection on the first principal plane.

- Les résultats du calcul des distances (théorème de Pythagore) entre les positions des points correspondant aux différentes moyennes réduites, dans l'espace à 3 dimensions défini par les axes des 3 composantes principales, peuvent être représentés sous forme d'un arbre hiérarchisé (fig. 4). La plus grande distance $(1,42)$ est obtenue pour le mode d'entretien du sol entre la position moyenne du désherbage et celle de l'enherbement $[1,42+/+0,566 /+/-0,754 /]$; ensuite vient l'espacement sur le rang $(0,64)$, puis l'écartement entre rangs $(0,62)$ et enfin le plan de comptage $(0,43)$.

Il faut remarquer que les rapports des distances les plus grandes pour les écartements $(2,60 / 1,80=1,44)$ et pour les espacements $(1,50 / 1,10=1,36)$ sont voisins dans l'essai, tout comme le sont ceux obtenus pour les racines, tant pour les espacements $(0,64)$ que pour les écartements $(0,62)$. Les résultats phytotechniques enregistrés depuis plusieurs années (REMOUE, 1978) montrent qu'il n'en est pas de même pour la partie aérienne, car les contraintes de palissage limitent le volume aérien disponible du cep, contraire- ment aux racines pour lesquelles il peut y avoir interpénétration entre souches voisines.

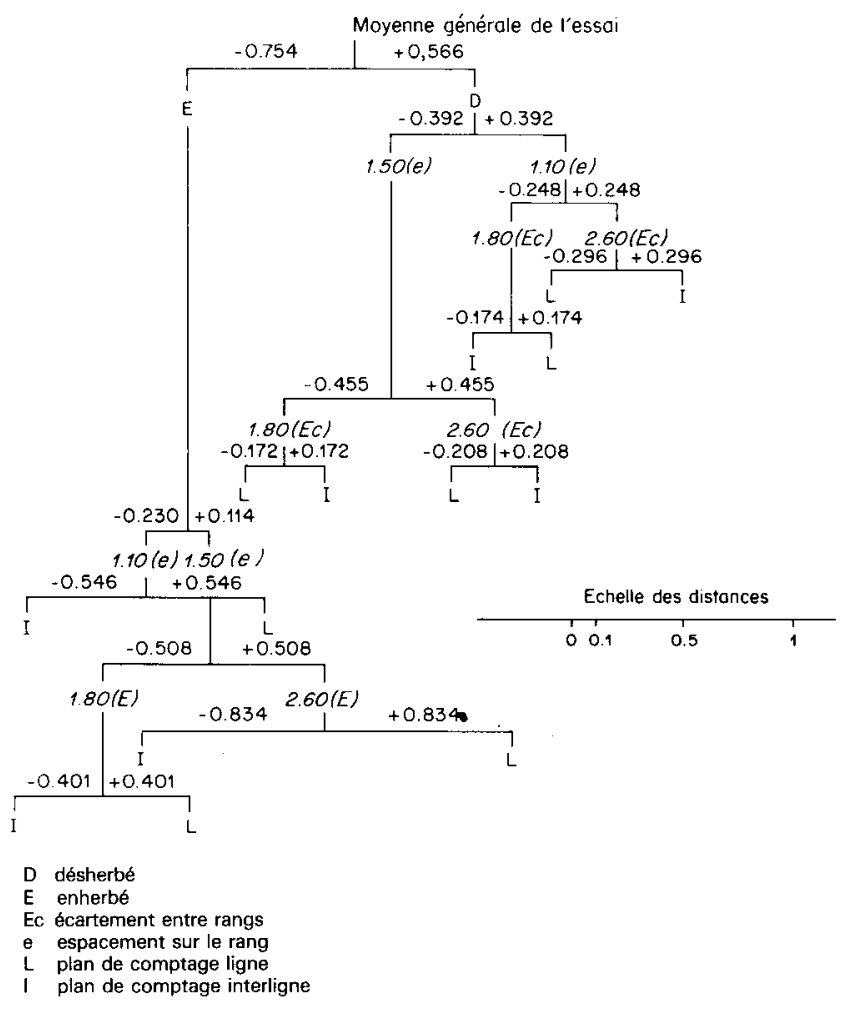

Figure 4

Arbre hiérarchisé des distances entre les positions dans l'espace des trois composantes principales des points moyens.

Hierarchical distances of mean points on three principal components.

\section{DISCUSSION}

La hiérarchisation de l'influence des facteurs faite grâce à l'analyse multidimensionnelle montre que le mode d'entretien du sol a un effet plus important que ceux, pratiquement identiques, de l'espacement et de l'écartement.

Les racines les plus fines (diamètre inférieur à $1 \mathrm{~mm}$ ) ne sont pas influencées significativement par la densité de plantation, tandis que l'horizon pédologique joue un rôle important (augmentation du nombre de racines en surface). Ces racines seraient plus sensibles aux conditions de milieu qu'à celles du peuplement et se développeraient plus dans les horizons enrichis. L'influence de l'horizon augmente avec l'espacement et la distance à la souche, ce qui laisserait supposer que le phénomène de compétition, qui théoriquement devrait la diminuer, se produirait logiquement, d'abord sur la ligne de plantation et en fortes densités.

Les faibles espacements augmentent significativement le nombre de racines de diamètre $1-2 \mathrm{~mm}$, tandis que les petits écartements $(1,80 \mathrm{~m})$ le diminuent.

L'effet de la densité de plantation n'est donc pas simple et ne peut être caractérisé qu'en le décomposant en espacement et écartement. Dans les conditions de l'essai, les influences de ces 2 facteurs sont contraires. Il en résulte que le traitement $1,80 \mathrm{~m} \times$ $1,50 \mathrm{~m}$ qui, sur le plan de la géométrie de la plantation semble le plus satisfaisant pour la colonisation 
du sol, est en fait le plus défavorable. Ces résultats ne transparaissent pas des travaux antérieurs (HIDAlGo \& CANDELA, 1966, 1969), mais ceux-ci s'appuyaient sur des essais à densité constante ou des plantations au carré. Certaines études même (BRANAS \& VERGNES, 1957) relatent des résultats opposés aux nôtres.

Les vignes larges ont un système racinaire plongeant plus développé que les vignes traditionnelles. Cela semble dû à une plus grande vigueur des souches (fig. 5). Ce mode de conduite permet une meilleure

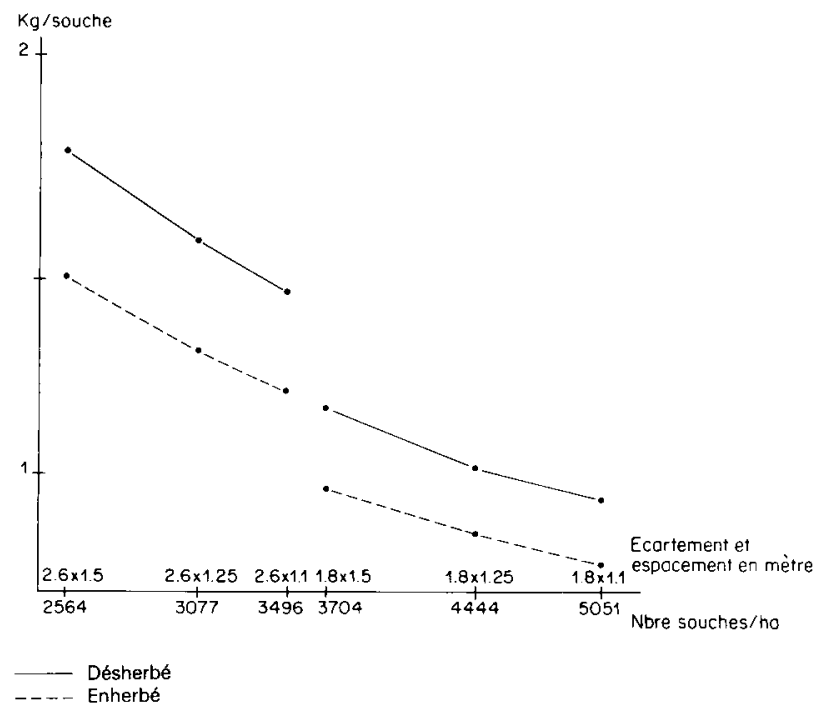

Figure 5

Moyennes de poids de bois de taille obtenu entre 1974 et 1982. Means of cane mass ( $\mathrm{kg} / \mathrm{vine}$ ) for nine successive seasons (19741982).

extraction de l'eau des horizons profonds si, comme dans les conditions de notre essai, le milieu édaphique ne présente aucune contrainte majeure à faible profondeur (REMOUE et al., 1981).

Les fortes densités augmentent le nombre de racines en surface, alors que les faibles densités l'accroissent en profondeur.

Il est probable que les vignes larges constituent un peuplement très différent des vignes traditionnelles au plan biologique et il n'existe pas de « continuum » de comportement dans la gamme de densités étudiée.

Si on adjoint une compétition inter-espèces (enherbement permanent - vigne) à la compétition intraspécifique, il y a une modification profonde du développement de l'enracinement. Le ray-grass diminue le nombre de racines dans l'interligne et surtout en surface, tandis que, dans les vignes larges, il favorise les racines plongeantes.

Dans cet essai, le développement du système racinaire est en relation étroite avec la vigueur. La moyenne des résultats obtenus entre 1974 et 1982 pour le poids de bois de taille par souche (fig. 5) permet de constater 2 familles de courbes :

- une $1^{\text {re }}$ famille comprenant les vignes larges $(2,60 \mathrm{~m} \times 1,50 \mathrm{~m} ; 2,60 \mathrm{~m} \times 1,25 \mathrm{~m} ; 2,60 \mathrm{~m} \times$ $1,10 \mathrm{~m}$ ) avec une décroissance régulière de la vigueur lorsque la densité croît.

- une $2^{\mathrm{e}}$ famille fortement décrochée par rapport à la première et comportant les vignes traditionnelles $(1,80 \mathrm{~m} \times 1,50 \mathrm{~m} ; 1,80 \mathrm{~m} \times 1,25 \mathrm{~m} ; 1,80 \mathrm{~m} \times$ $1,10 \mathrm{~m})$, avec une pente plus faible.

L'enherbement décale de façon notable les 2 familles de courbes en exerçant une influence défavorable sur la vigueur. La densité $1,80 \mathrm{~m} \times 1,50 \mathrm{~m}$, très proche de la plantation $2,60 \mathrm{~m} \times 1,10 \mathrm{~m}$ sur le plan de nombre de souches à l'ha (3 704 souches contre 3 497), entraîne une diminution considérable de la vigueur pouvant être reliée à un enracinement moins développé.

Reçu le 2 juin 1983 Accepté le 30 janvier 1984

\section{RÉFÉRENCES BIBLIOGRAPHIQUES}

Branas J.. Vergnes A., 1957. Morphologie du système radiculaire. Prog. Agric. Vitic., 29, 32, 146-151.

Dagnelie P., 1975. Théorie et méthodes statistiques. T. 2, Presses agronomiques de Gembloux, $463 \mathrm{p}$.

Hidalgo L., Candela M. R., 1966. Influencia de la densidad y disposicion de plantacion en la produccion del vinedo. Inst. Nac. Investig. Agron. Madrid, $68 \mathrm{p}$.

Hidalgo L., Candela M. R., 1969. Morfologia radicular de la Vid, su relacion con la produccion, densidad y disposicion del Vinedo. Inst. Nac. Investig. Agron. Madrid, 101 p.

Laurent G., 1979. Etude chez la vigne, en conditions naturelles, de l'influence de la densité de plantation et du palissage sur le développement des racines et des radicelles, Mémoire ENITA Bordeaux, $73 \mathrm{p}$.

Morlat R., 1981. Effets comparés de deux techniques d'entretien du sol sur l'enracinement de la vigne et sur le milieu édaphique. Agronomie, 1 (10), 887-896.
Pinet P., 1980. Influence de la densité de plantation et du mode d'entretien du sol sur le système racinaire d'un peuplement de vigne et sur quelques propriétés physico-chimiques du sol. Mémoire ENITA Bordeaux, 31 p. + annexes.

Radoulov L., Braikov D., 1974. Développement du système radiculaire chez la vigne en fonction de la densité de plantation et du procédé de formation. Sadovod, Vinograd, Vinodel (Moldavie), URSS, 5, 29, 61-63.

Remoué M., 1978. Que peut-on espérer des nouveaux modes de conduite de la vigne; perspectives d'avenir apportées par les expérimentations. Rev. Fr. OEnol. 71, 17-21.

Remoué M., Morlat R., Pinet P., 1981. Influence de la densité de plantation et du mode de conduite sur le développement et les caractéristiques d'un peuplement de vigne dans le Val de Loire. Mémoire ATP "Compétition intraclonale chez les végétaux ligneux », $11 \mathrm{p} .+$ annexes.

Schatouiro L. F., Alief N. A., Gadjiev Z. Sch., Gasanov A.A., 1978. Développement du système radiculaire chez les vignes à rang large et à haute tige. Vinodel. Vinograd. SSSR 5, 340, 19-43. 